\title{
Computing Symmetries of Combinatorial Objects (Abstract)
}

\author{
Brendan D. McKay \\ Department of Computer Science, \\ Australian National University \\ bdm@cs.anu .edu. au
}

We survey the practical aspects of computing the symmetries (automorphisms) of combinatorial objects. These include all manner of graphs with adornments, matrices, point sets, etc.. Since automorphisms are just isomorphisms from an object to itself, the problem is intimately related to that of finding isomorphisms between two objects. 